\section{AB0134 BLOOD LYMPHOCYTE SUBSETS ACCORDING TO THE CLINICAL PROFILE IN SJÖGREN'S SYNDROME}

F. Barcelos ${ }^{1,2,3}$, C. Martins ${ }^{3}$, G. Nunes ${ }^{3}$, T. Lopes ${ }^{3}$, J. Vaz Patto ${ }^{1}$, J. Amaral ${ }^{4}$ J.C. Branco ${ }^{3}$, L.M. Borrego ${ }^{3} .1$ Instituto Português Reumatologia; ${ }^{2}$ Hospital CUF Descobertas; ${ }^{3}$ Nova Medical School; ${ }^{4}$ Faculdade Med Dentária, UL, Lisboa, Portugal

Background: It has been recently described the occurrence of disturbances in lymphocyte subsets in Sjögren's syndrome (SS) which may reflect B cell hyper activation and a T cell adjuvant role.

Objectives: We aim to characterize circulating lymphocyte subsets in SS patients, according to disease activity and antibody profile.

Methods: We have included in this study 53 SS patients (2002 AECG criteria) of which 22 with $>10$ years since diagnosis and 31 with $<2$ years since diagnosis, and 22 healthy controls. Lymphocyte subsets, including follicular (Tfh) and regulatory $\mathrm{T}$ (Treg) cells, maturation subsets, plasmablasts $(\mathrm{PB})$ and regulatory B (Breg) cells, were characterized by flow cytometry. Statistical analysis was performed with GraphPad. Significance was considered for $p<0.05$.

Results: Compared to controls, SS patients had lower absolute counts of $B$ $(p=0.0337)$ and T cells $(p=0.0012)$, lower CD4 $(p=0.0002)$ and higher CD8 percentages $(p=0.0006)$, resulting in an increased CD4/CD8 ratio $(p=0.0006)$. Additionally, there was decrease in absolute counts of Tregs $(p=0.0008)$ and Th17 cells $(p=0.0005)$ in SS patients. Moreover, there was a decreased absolute counts $(\mathrm{p}<0.0001)$ of Tfh cells, identified by CXCR5 expression, though higher levels of IL2 $1^{+}$CD4 T cells $(p=0.0209)$ and Tfh1 cells $(p=0.0092)$. SS patients also presented higher $\%$ of naïve B cells $(p=0.0412)$, lower $\%$ and absolute counts of memory $(\%, p=0.0161$; abs $p=0.0002)$ and unswitched memory $(\%, p=0.0106$; abs $p=0.0005) B$ cells and lower absolute counts $(p=0.0001)$ of switched memory $B$ cells, with higher naive/memory $B$ cell ratios, compared to healthy subjects $(p=0.0219)$. Accordingly, using the $B m 1-5$ classification, we have found decreased $\mathrm{Bm} 1(\%, p=0.0087$; Abs, $p=0.0007$ ), eBm5 (Abs, $p=0.0005$ ) and $B m 5$ cells (Abs, $\mathrm{p}=0.0015)$ in SS patients. Similar Bm2+Bm2'/eBm5+Bm5 ratios were observed in patients and controls. CD24 ${ }^{+} \mathrm{CD} 27^{+}$Bregs were also decreased $(p \leq 0.0012)$ in SS patients.

SS patients had also an increase in IL $21^{+} \mathrm{CD} 4 \mathrm{~T}$ cells, particularly in patients with extra-glandular manifestations (EGM) $(n=12)$, who also presented less Tfh17 cells $(p=0.0409)$ comparing to patients without EGM. PB were decreased in patients with EGM only when compared to controls $(\mathrm{p}=0.0434)$.

$\mathrm{SSA}^{+}$patients $(\mathrm{n}=36)$ had more frequently EGM than $\mathrm{SSA}^{-}$, higher ESSDAI score, $\gamma$-globulin levels and lower salivary flow. Compared to SSA and controls, increased IL21+CD4 T cells were detected in SSA ${ }^{+}$patients $(p \leq 0.0284)$, who also had higher Tfh1 cells than controls $(p \leq 0.0065)$. Lower Bm1 cells $(p \leq 0.0305)$ were observed in SSA $\mathrm{S}^{+}$atients compared to SSA $\mathrm{A}^{-}$and controls, with an increase $\%$ of Bm3+Bm4 cells $(p=0.011)$ compared to SSA ${ }^{-}$

Conclusions: The immune profile of SS patients has distinctive features, with decreased memory B cell subsets, including CD $24^{+} \mathrm{CD} 27^{+}$Bregs. Moreover, an increased capacity of IL21 secretion by T cells, together with a more prominent Tfh1 signature seem to be the hallmark of SS, particularly in patients with EGM and autoantibody production, suggesting an immune environment prone to proinflammatory processes, in severe patients. Comprehending the immune dynamics in SS may be valuable for diagnosis, follow-up and future therapeutic decisions.

Disclosure of Interest: None declared

DOI: 10.1136/annrheumdis-2017-eular.3030

\section{AB0135 PLASMATIC AND URINARY ENDOTHELIAL MICROPARTICLES ARE INCREASED IN PATIENTS WITH LUPUS NEPHRITIS}

F. Miranda, C. Barbati, S. Truglia, F.R. Spinelli, F. Ceccarelli, C. Alessandri, G. Valesini, F. Conti. Dipartimento di Medicina Interna e Specialità Mediche, Reumatologia, Sapienza Università di Roma, Rome, Italy

Background: Systemic lupus erythematosus (SLE) is a chronic autoimmune disease presenting with a wide array of clinical manifestations and incompletely understood pathogenesis. SLE is characterized by alterations in both the innate and adaptive immune system ultimately leading to the loss of immunologic tolerance and occurrence of autoantibodies against nuclear material. Lupus nephritis is one of the most severe features of SLE determining an increase in morbidity e mortality rates. Renal biopsy still represent a fundamental diagnostic and prognostic tool for LN. Therefore, non-invasive surrogate biomarkers of active LN are urgently needed. Circulating, heterogeneous subcellular microparticles (MPs) are released from cells and platelets constitutively and upon cellular activation or apoptosis. Such MPs may reflect the state of their parental cells and tissues, and could serve as markers of pathology. Particularly in SLE, MPs are potential biomarkers and triggers of autoimmunity. Recent studies have demonstrated increased levels of plasmatic EMPs in patients with SLE active disease and their reduction after treatment.

Objectives: The aim of this study was to investigate plasmatic and urinary levels of endothelial microparticles in a cohort of SLE patients with and without renal involvement compared to healthy controls.

Methods: Consecutive SLE patients and sex- and age-matched $\mathrm{HC}$ were included in the study. MPs were isolated from plasma and urine and characterized by flow cytometry using AnxV (a probe that binds to the exposed phosphatidilserine
-PS) and antibodies against surface markers endothelial cells (CD31+CD41-). Mann-Whitney and Spearman correlation tests were used. A $p$ value $<0.05$ was considered statistically significant.

Results: Sixty SLE patients (55F:5M, age 41,7 \pm 9.6 Y disease duration $149 \pm 112$ months) and 29 healthy controls were studied. Twenty-eight patients had renal involvement.The total number of plasmatic MPs was lower in SLE patients than $\mathrm{HC}(\mathrm{p}=0.001)$. In contrast there was no significant difference in levels of EMPs between the two groups. When the patients were divided according to renal involvement, the patients with active lupus nephritis (A-LN) showed lower plasmatic level of EMPs in comparison to inactive LN (I-LN) $(p=0.01)$, while the patients with I-LN had higher levels of EMPs than HC $(p=0.002)$. There was no significant difference of total urinary level of MPs between SLE patients and HC. Urinary levels of EMPs were higher in SLE and in lupus nephritis patients than HC.

Conclusions: The results of the present study show increased urinary and plasmatic levels of EMPs in patients with lupus nephritis in remission. Circulating EMPs have been considered as a potential biomarker of endothelial activation and damage in several autoimmune disorders, and higher EMP levels have been detected in patients with vasculitis and associated with disease activation. According to our results, plasmatic EMPs levels are higher in inactive LN patients than in healthy donors. These results may suggest a potential role of EMP as a biomarker of lupus nephritis

Disclosure of Interest: None declared

DOI: 10.1136/annrheumdis-2017-eular.5003

\section{AB0136 MICROPARTICLES FROM SLE PATIENTS ARE A SOURCE OF INTERFERON-ALPHA}

F. Miranda, C. Barbati, F.R. Spinelli, F. Ceccarelli, S. Truglia, C. Alessandri, G. Valesini, F. Conti. Dipartimento di Medicina Interna e Specialità Mediche, Reumatologia, Sapienza Università di Roma, Rome, Italy

Background: Systemic lupus erythematosus (SLE) is the prototype of systemic autoimmune disorders. In the late 1970s, increased serum levels of interferon (IFN) were shown for the first time to be significantly associated with SLE and to correlate with disease activity. IFN $\alpha$ is a pleiotropic cytokine that can affect multiple cell types involved in lupus.Plasmacytoid dendritic cells have a special role in the production of IFN and are the main sources of serum interferon. IFN has the potential to dramatically influence the development, progression, and pathogenesis of SLE as it can influence the function and activation state of most major immune cell subsets and function as a bridge between innate and adaptive immunity.Lupus-prone mouse models, indicates that the type I interferon system may play a pivotal role in the pathogenesis of several lupus and associated clinical features, such as nephritis, neuropsychiatric and cutaneous lupus.Circulating microparticles (MPs) are ubiquitous in the blood of healthy individuals, These MPs play an active role in coagulation and intercellular communication and assist in activation or suppression of the immune system, depending on their parental cell origin. Changes in the concentration and/or composition of circulating microparticles have been described in various autoimmune diseases, including rheumatoid arthritis (RA) systemic sclerosis (SSc) and systemic lupus erythematosus (SLE). For SLE, the reported microparticle-related changes remain somewhat inconclusive.

Objectives: To better understand the role of MPs in SLE patients, we analyzed the presence of interferon alpha on MPs surface.

Methods: MPs were isolated from citrate-treated plasma; blood cells were removed by two steps of centrifugation process $(2500 \mathrm{~g}$ for $15 \mathrm{~min}$ at $20 \mathrm{C}$ two time). The resulting platelet-poor-plasma (PPP), was analyzed by flow cytometry with specific antibody against IFN alpha

Results: 20 consecutive SLE patients (10 with active lupus nephritis) and 10 sex- and age-matched healthy control subjects were included in the study. We found that MPs from SLE patients carry on their surface IFN alpha.Moreover, the percentage IFNalpha + MPs was higher in SLE patients and in lupus nephritis patients than in $\mathrm{HC}$, but there was not significant difference between patients with and without renal involvement.

Conclusions: The results of the present study show for the first time the presence of IFN alpha on MPs surface. We may assume that INF+ MPs derive from dendritic cells. In lupus nephritis patients the increased recruitment of dendritic cells was at tubular interstitial level, with subsequent IFN alpha production. Interestingly, MPs (containing RNA and DNA) could stimulate type I IFN production in plasmacytoid dendritic cells and MPs releasing.

Disclosure of Interest: None declared

DOI: 10.1136/annrheumdis-2017-eular.5171

\section{AB0137 ALTERATIONS IN MICRORNA EXPRESSION PROFILES IN PRIMARY SJÖGREN'S SYNDROME AND SYSTEMIC LUPUS ERYTHEMATOSUS}

G. Papp ${ }^{1}$, J.-Q. Chen ${ }^{1}$, S. Póliska ${ }^{2}$, K. Szabó ${ }^{1}$, T. Tarr ${ }^{1}$, B.L. Bálint ${ }^{2}$, P. Szodoray ${ }^{3}$, M. Zeher ${ }^{1} .{ }^{1}$ Division of Clinical Immunology; ${ }^{2}$ Department of Biochemistry and Molecular Biology, University of Debrecen; ${ }^{3}$ Centre for Immune Regulation, University of Oslo, Debrecen, Hungary

Background: MicroRNAs (miRNAs) are single-stranded, endogenous non- 
coding small RNAs, ranging from 18 to 25 nucleotides in length. MiRNAs are essential in regulating gene expression, cell development, differentiation and function. Dysregulation in miRNAs expression may contribute to the development of autoimmunity. However, a given miRNA may have hundreds of different mRNA targets and a target might be regulated by multiple miRNAs, thus the characterisation of dysregulated miRNA expression profiles could give a better insight into the development of immunological disturbances in autoimmune diseases.

Objectives: The aim of our study was to examine the changes in miRNA expression profiles in patients with primary Sjögren's syndrome (pSS) and systemic lupus erythematosus (SLE).

Methods: Eight pSS patients, 8 SLE patients and 7 healthy control subjects were enrolled in the investigation. MiRNAs were isolated from peripheral blood mononuclear cells, and expression patterns were determined with Illumina next-generation sequencing technology. Since the immunopathogenesis of pSS and SLE encompasses pronounced B cell hyperactivity along with specific autoantibody production, we paid a special attention on the association between miRNA expression levels and altered peripheral B cell distribution.

Results: In SLE patients 135, while in pSS patients 26 miRNAs showed altered expression. Interestingly, the 25 miRNAs including miR-146a, miR-16 and miR21 , which were over-expressed in pSS patients, were found to be elevated in SLE group, as well. On the contrary, we observed the down-regulation of miR-150-5p, which is a novel and unique finding in pSS. Levels of several miRNAs over-expressed in SLE, were not changed in pSS, such as miR-148a3p, miR-152, miR-155, miR-223, miR-224, miR-326 and miR-342. Expression levels of miR-223-5p, miR-150-5p, miR-155-5p and miR-342-3p, which miRNAs are potentially linked to B cell functions, showed associations with the B cell proportions within peripheral blood mononuclear cells.

Conclusions: The observed differences in miRNA expression profiles and the better understanding of immune regulatory mechanisms of miRNAs may help to elucidate the pathogenesis of pSS and SLE.

Acknowledgements: This work was supported by the ÚNKP-16-4-III New National Excellence Program of the Ministry of Human Capacities.

Disclosure of Interest: None declared

DOI: 10.1136/annrheumdis-2017-eular.3205

\section{AB0138 INTERFERON-GAMMA CHALLENGE OF PBMC FROM PATIENTS WITH LUPUS NEPHRITIS IN REMISSION DECREASES SUPPRESSOR OF CYTOKINE SIGNALING 1 (SOCS1) AND REGULATORY T CELLS (TREGS) AND PROMOTES IMMUNE ACTIVATION}

G. Gibor ${ }^{1}$, U. Arad ${ }^{1}$, J. Wallman ${ }^{1}$, J. Ablin ${ }^{1}$, V. Aloush ${ }^{1}$, I. Kaufman ${ }^{1}$, S. Jacky ${ }^{2}$, D. Caspi i ${ }^{1}$, O. Elkayam ${ }^{1}$, D. Paran ${ }^{1}$, A. Sharabi ${ }^{3} .{ }^{1}$ Rheumatology; ${ }^{2}$ Tel Aviv Medical Center, Tel Aviv, Israel; ${ }^{3}$ Harvard BIDMC, Boston MA, United States

Background: Interferon-gamma (IFN- $\gamma$ ) plays an important role in the development of lupus nephritis (LN). Regulation of IFN- $\gamma$ signaling that occurs in disease remission and in active $L N$ is herein addressed.

Objectives: To study the impact of IFN- $\gamma$ on PBMC obtained from patients with LN in remission as compared to active LN.

Methods: Sixteen patients fulfilling the ACR classification criteria for systemic lupus erythematosus were recruited. All patients had a history of $L N$ of whom 10 were in remission (as defined by EULAR criteria) and 6 had active LN (as defined by SLEDAI-2K or BILAG). Healthy subjects $(n=10)$ were included as a control group. Sera and PBMC were obtained from each individual. Flow cytometry, western blots and real time RT-PCR were used in processing and detection of cell subtypes, protein and mRNA levels. Recombinant human IFN- $\gamma($ rhIFN- $\gamma$ ) and anti-IFN- $\gamma$ neutralizing antibody were used in vitro. Mann-Whitney and student t-tests were used for statistical analysis.

Results: In active LN there was a significant 2-fold increase in $\mathrm{CD} 4^{+} \mathrm{CD} 69^{+}$ activated $\mathrm{T}$ cells as compared to healthy subjects and patients in remission. Reactivity to interferon-gamma receptor was determined by the phosphorylation of its predominant transcription factor, signal transducer and activator of transcription 1 (STAT1) in the cells that were incubated with rhIFN- $\gamma$ or with media alone. In active LN, 3- and 6-fold increase in pSTAT1 occurred with rhIFN- $\gamma$ incubation during $24 \mathrm{~h}$ and $48 \mathrm{~h}$, respectively, and healthy subjects responded likewise. In patients in remission, pSTAT1 increase was even higher (by 8- and 10-fold at 24h and $48 \mathrm{~h}$, respectively). After $24 \mathrm{~h}$ incubation with rhIFN-g all groups had elevated (mRNA) expression of SOCS1, but at $48 \mathrm{~h}$, it significantly decreased in healthy subjects and in patients in remission by $34 \%$ and $50 \%$, respectively. Further, at $24 \mathrm{~h}$ the frequency of $\mathrm{CD}^{+} \mathrm{CD} 127^{\text {low }} \mathrm{FoxP}^{+}$regulatory $\mathrm{T}$ cells (e.g. Tregs) increased by $27-30 \%$ in active LN and in healthy subjects, and in remission it was minimally changed. At $48 \mathrm{~h}$, the frequency of Tregs significantly decreased in healthy subjects (to baseline levels before rhIFN- $\gamma$ challenge) and in patients in remission (by $24 \%, p=0.003$ ). A challenge of PBMC from $L N$ patients in remission with sera (at $1 \%$ concentration) derived from patients with active LN resulted in 7.5-fold increase in PSTAT1 expression and 20-30\% decrease in Tregs, however, combination of sera and anti-IFN-g neutralizing antibody resulted in 5-fold decrease in PSTAT1 expression and significantly diminished the decrease in Tregs.

Conclusions: In LN in remission a challenge with IFN- $\gamma$ could lead to immune activation and a risk of flare-up, as it results in a decrease in both SOCS1 and
Tregs and a robust STAT1 phosphorylation. In active LN, STAT1 phosphorylation is less diminishing, as both SOCS1 and Tregs are saturated, which could affect their suppressive effectiveness.

Disclosure of Interest: None declared

DOI: 10.1136/annrheumdis-2017-eular.4281

\section{AB0139 ROLE OF MUCOSAL-ASSOCIATED INVARIANT T (MAIT) CELLS} IN A LUPUS MODEL

G. Murayama ${ }^{1,2}$, A. Chiba ${ }^{2}$, A. Nomura ${ }^{2}$, H. Amano ${ }^{1}$, K. Yamaji ${ }^{1}$, N. Tamura ${ }^{1}$, S. Miyake ${ }^{2} \cdot{ }^{1}$ Department of Internal Medicine and Rheumatology; ${ }^{2}$ Department of Immunology, Juntendo University School of Medicine, Tokyo, Japan

Background: Mucosal-associated invariant T (MAIT) cells are innate T cells that are restricted by MHC-related molecule-1 (MR1) and express a semi-invariant TCR $\alpha$ chain: V $\alpha 7.2-J \alpha 33$ in humans and $V_{\alpha} 19-J \alpha 33$ in mice. Previously, we have demonstrated that MAIT cells played a protective role against experimental autoimmune encephalomyelitis, an animal model of human multiple sclerosis. We found that MAIT cells are activated in patients with systemic lupus erythematosus (SLE) and that the activation state of MAIT cells correlated with SLE disease activity index (SLEDAI) score, suggesting their association in lupus pathology. Objectives: We set out to clarify functions of MAIT cells in a lupus model by using $\mathrm{F}_{\mathrm{C}} \gamma \mathrm{RIIB}^{-/-}$Yaa mice.

Methods: Fc $\gamma$ RIIB $^{-/}$Yaa mice were crossed to MR1 deficient mice lacking MAIT cells, and disease progression was compared between MR1 ${ }^{-/} \mathrm{Fc} \mathrm{RIIB}^{-1-}$ Yaa and MR1 ${ }^{+/+} \mathrm{Fc} \gamma \mathrm{RIIB}^{-1-}$ Yaa mice at 1-4 months of age. Serum anti-dsDNA antibody levels were measured and urinary microalbumin were evaluated. At the time of sacrifice, at 4 months of age, the severity of nephritis and dermatitis were assessed by histologically and IgG deposition in skin and glomeruli was measured.

Results: Survival rate was significantly reduced in $\mathrm{MR}^{-1} \mathrm{Fc} \gamma \mathrm{R} / \mathrm{ll} \mathrm{b}^{-/}$Yaa mice compared with MR1//+ FcyRllbb-/- Yaa mice. Anti-dsDNA antibody levels were remarkably higher in $\mathrm{MR}^{+/+}$than $\mathrm{MR} 1^{-/} \mathrm{Fc} \gamma \mathrm{R} / \mathrm{ll}{ }^{-/}$Yaa mice at 4 months of age. Even though Glomeruli were significantly enlarged both in $\mathrm{MR} 1^{+/+}$and $\mathrm{MR}^{-1-} \mathrm{Fc} \gamma \mathrm{RIIb}^{-1-}$ Yaa mice due to a marked cellular proliferation in glomeruli, the glomerulonephritis score tended to be lower in $\mathrm{MR}^{-/} \mathrm{Fc} \gamma \mathrm{Rllb}{ }^{-/}$Yaa mice compared with $\mathrm{MR}^{+/+} \mathrm{Fc} \gamma \mathrm{R} / \mathrm{ll} \mathrm{b}^{-1-}$ Yaa mice. A larger amount of IgG deposition was observed in mesangial area and along glomerular capillary walls in

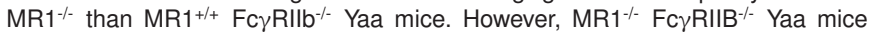
showed exacerbated inflammation in the skin lesions. There was a high degree of inflammatory cells infiltration into the skin and a significant worsening of dermatitis

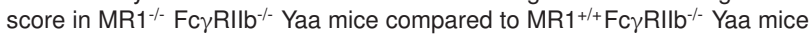
Conclusions: These data suggests that MAIT cells exhibit dual roles in lupus pathogenesis. MAIT cells enhance autoantibody production and the disease severity of nephritis, but have a suppressive effect on dermatitis. Further studies are under going to uncover the mechanisms by which MAIT cells are involved in each target tissues.

References:

[1] Croxford JL, et al. Invariant V(alpha)19i T cells regulate autoimmune inflammation. Nat Immunol. 2006; 7(9): 987-94.

[2] Chiba A, et al. Mucosal-associated invariant $T$ cells promote inflammation and exacerbate disease in murine models of arthritis. Arthritis Rheum. 2012; 64(1): 153-61.

[3] Kawano S, et al. Phenotype conversion from rheumatoid arthritis to systemic lupus erythematosus by introduction of Yaa mutation into Fc $\gamma$ RIIIB-deficient C57BL/6 mice. Eur J Immunol. 2013; 43(3): 770-8.

Disclosure of Interest: None declared

DOI: 10.1136/annrheumdis-2017-eular.3086

\section{AB0140 LUPUS-PRONE SLAM HAPLOTYPE EXERTS MONOCYTOSIS AND DEVELOPS SPECIFIC PHENOTYPE OF AUTOIMMUNE DISEASE INTRODUCED BY YAA MUTATION}

H. Amano ${ }^{1}$, K. Nishikawa ${ }^{2}$, Q. Lin ${ }^{3}$, S. Kawano ${ }^{1}$, K. Yamaji ${ }^{1}$, S. Hirose ${ }^{3}$, N. Tamura ${ }^{1} .{ }^{1}$ Department of Internal Medicine and Rheumatology; ${ }^{2}$ Department of Pathology, Juntendo University School of Medicine, Tokyo; ${ }^{3}$ Department of Biomedical Engineering, Toin Human Science and Technology Center, Toin University of Yokohama, Yokohama, Japan

Background: We previously obtained a 129-derive Fc $\gamma R$ RIIB-deficient C57BL/6 (B6) congenic strain of mice, which spontaneously developed severe rheumatoid arthritis (RA) ${ }^{1}$. The introduction of the Yaa (Y-linked autoimmune acceleration) mutation, which is a consequence of a translocation from the telomeric end of the $\mathrm{X}$ chromosome containing the TIr7 gene onto the $\mathrm{Y}$ chromosome, to the Fc $\gamma$ RIIIB-deficient B6 mice (B6.Fc $\gamma$ RIIB-/.. Yaa) developed lupus like nephritis but not $\mathrm{RA}^{2}$.

Objectives: By extensively backcrossing 129-based Fc $\gamma$ RIIIB-deficient mice to B6 mice, we established wildtype Fc $\gamma R$ RIIB and 129-derive autoimmune-prone SLAM haplotype $\left(S / a m^{129}\right)$. We examined the phenotype of Slam ${ }^{129}$ mice, and also $S^{\prime a m}{ }^{129}$. Yaa mice by introducing Yaa mutation to these mice.

Methods: We analyzed peripheral blood monocyte subset and also serum autoantibodies as well as immunohistopathological findings of kidneys and lungs. Results: Slam ${ }^{129}$ mice showed age-associated monocytosis with marked expan- 